\title{
Clinico-pathological Conference
}

\section{Spinal cord disease due to vacuolar myelopathy in AIDS}

\author{
R F Miller, B Sweeney, M J G Harrison, S B Lucas
}

\section{Case Reports (Dr R F Miller) \\ Case 1:}

A 43 year old non-smoking Caucasian homosexual teacher was found to be HIV-1 antibody positive and hepatitis B immune in April 1989. At this time he had chronic stable angina, treated with a B blocker and oral nitrates, and recurrent diarrhoea passing one to four liquid stools per day. Giardia lambia was isolated from stool and his symptoms resolved with metronidazole treatment. In June 1990 he began primary prophylaxis against pneumocystis pneumonia with monthly inhaled pentamidine. At this time his angina had deteriorated significantly and so he underwent coronary artery bypass grafting in July of that year. In September 1990 he represented to the genitourinary clinic with persistent oral candidiasis and a large ulcer on the right fauces: swabs from this area were negative for cytomegalovirus and Herpes simplex virus and his CD4 count was $90 / \mathrm{mm}^{3}$ (normal $=350-2,200 / \mathrm{mm}^{3}$ ). Treatment with Difflan oral rinse and hydrocortisone pellets and thalidomide was tried without benefit. Biopsy of the ulcer showed only changes typical of aphthous ulceration. Oral prednisolone and carbenoxalone gel were given and the ulcer began to heal. In December 1990 zidovudine was begun.

The patient became dysphonic in January 1991 and was found to have palatal incompetance secondary to fibrosis caused by healing of the ulcer. In March of that year the ulcer recurred and was re-biopsied. Histology this time showed a high grade B cell lymphoma. Staging investigations included a bone marrow aspirate and trephine and CT of the base of the skull; both were negative. Radiotherapy was given to the throat, $30 \mathrm{~Gy}$ in 15 fractions. Chronic diarrhoea had developed during this time and cryptosporidium was identified in stool and duodenal biopsy. Azithromycin and codeine phosphate rapidly controlled the diarrhoea. Liver function tests at this time were abnormal; alkaline phosphatase $=885$ (normal range $=100-280$ ) IU/1. Transaminase and bilirubin levels were within normal limits. An ultrasound showed a dilated common bile duct and intrahepatic ducts, consistent with a diagnosis of AIDS sclerosing cholangitis.

The patient's condition remained stable until September 1992 with variable diarrhoea, at this point he reported weight loss of $7 \mathrm{~kg}$, a worsening of his diarrhoea (passing up to 20 liquid stools per day) onset of dysphonia and right ear pain, a non-productive cough, and dysaesthesia in his feet. On examination he was pale and thin and had absent ankle jerks; plantar reflexes were flexor. Investigations showed $\mathrm{Hb}=8.6 \mathrm{~g} / \mathrm{dl}, \mathrm{WBC}=3.0 \times 10^{9} / 1$ ( $80 \%$ neutrophils). Microscopy and culture of stool revealed cryptosporidium and $\mathrm{Myco-}$ bacterium kansasii. An induced sputum sample contained Staphlococcus aureus, $M$ kansasii and Nocardia asteroides; blood cultures were negative. An MRI scan of the spine was normal and of the head showed minor cortical atrophy but there were no white matter signal changes suggestive of HIV encephalopathy. CSF analysis was normal save for a slightly raised protein, $0.5 \mathrm{~g} / 1$. Nerve conduction studies were performed.

\section{Dr B Sweeney}

The nerve conduction studies showed a mild generalised slowing of sensory and motor conduction velocities, with normal sensory and motor action potentials. These findings would be most compatible with a demyelinating, rather than an axonal neuropathy. However, since slowing of nerve conduction velocities has been described in patients with AIDS who do not have symptoms or signs of neuropathy the exact relevance of this finding is unclear. ${ }^{1}$ There was no clinical evidence of severe weakness, compatible with a demyelinating neuropathy. The patient had also been treated with thalidomide but this is associated with an axonal, rather than a demyelinating neuropathy.

\section{Dr R F Miller}

A chest radiograph suggested a left hilar mass, but CT of the thorax showed intrapulmonary consolidation only. A biopsy of the throat revealed post radiotherapy changes. $\mathrm{He}$ was given rifampicin, ethambutol and ciprofloxacin as treatment for $M$ kansasii; the rifampicin also being effective against nocardia and $S$ aureus. Zidovudine was discontinued and a blood transfusion was given. A trial of oral paromomycin (a non-absorbable aminogycoside) for cryptosporidia was of no benefit; his diarrhoea was initially controlled with a subcutaneous infusion of diamorphine via a syringe driver, subsequently with oral 
Figure 1 Case 1: Chest radiograph showing left hilar mass.

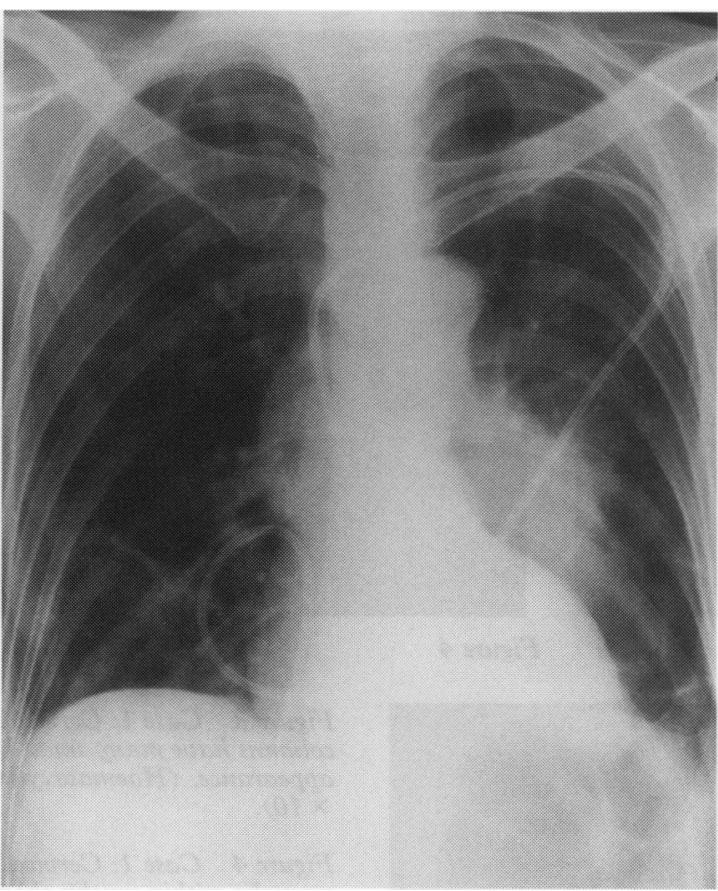

morphine. After six weeks in hospital the patient was discharged home without diarrhoea, his chest radiograph having cleared.

He was re-admitted three months later with a bacterial chest infection which was treated with oral antibiotics. He also reported a visual disturbance in his left eye; cytomegalovirus retinitis was diagnosed on the basis of typical retinal appearances. Ganciclovir was given via a Hickman line. A chest radiograph showed a large left hilar mass (fig 1). M kansasii was isolated from induced sputum and blood. The patient's condition deteriorated rapidly; he was transferred to a hospice and died. An autopsy was performed.

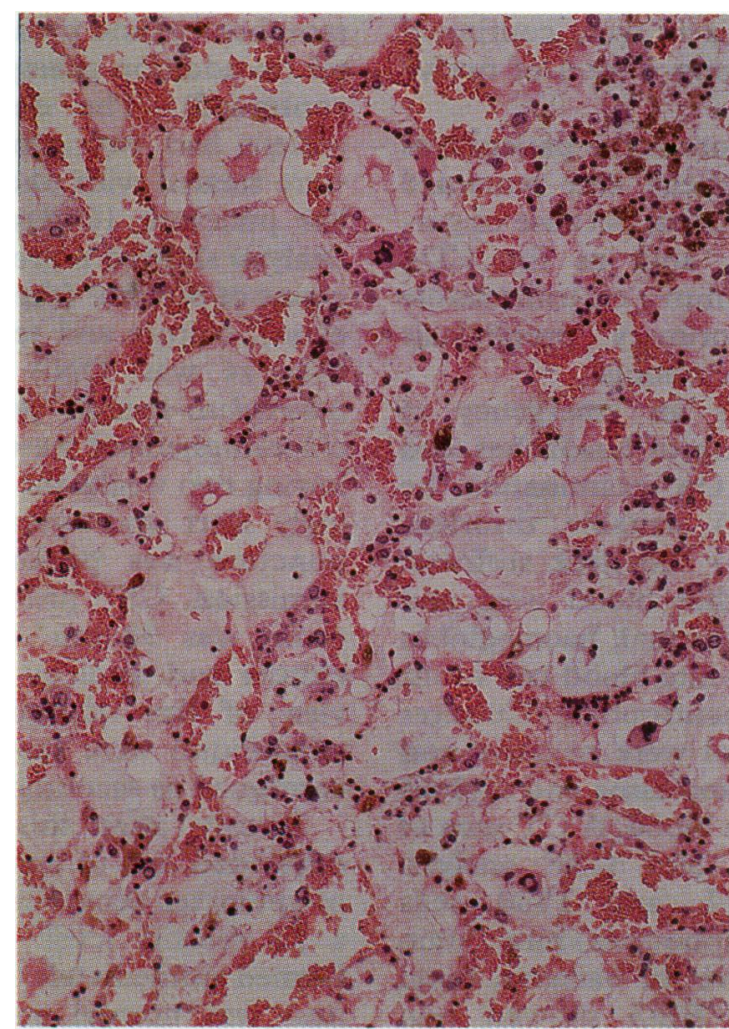

Autopsy (Dr S B Lucas)

The patient was wasted but not jaundiced. The heart had atheromatous coronary arteries and two vessel coronary artery bypass grafts which were patent. There was a necrotic left hilar mas which involved the right atrium. The left lung weighed $660 \mathrm{~g}$ and had $\mathrm{a}$ $6 \times 5 \times 4 \mathrm{~cm}$ necrotic white mass in the lower lobe; distal to this the lung was consolidated. Multiple white deposits 0.5 to $3 \mathrm{~cm}$ in diameter were evident in the liver (which was enlarged at $1680 \mathrm{~g}$ ), hilar nodes, perihepatic and mesenteric nodes. The common bile duct was dilated (circumference $4 \mathrm{~cm}$ ) and had a ragged mucosa. Bone marrow, spleen, spinal cord and brain (weight $1590 \mathrm{~g}$ ) were macroscopically normal. The nasopharynx was not examined.

Histopathology revealed a necrotic high grade B-cell lymphoma of the lung, liver, right atrium and lymph nodes. Within the left lung, distal to the lymphoma, was Pneumocystis carinii pneumonia; this was not present elsewhere in the lungs. The extra-hepatic bile ducts were inflamed with scanty cytomegalovirus inclusions in endothelial cells. No cryptosporidia were visible in the duct but they were plentiful in the adjacent duodenal mucosa. Zeihl-Neelsen stains showed foci of atypical mycobacteriosis in abdominal lymph nodes and the spleen. The marrow was severely atrophic, with gelatinous transformation (fig 2). The cerebrum had focal cytomegalovirus ependymitis and there was vacuolar myelopathy in the upper thoracic spinal cord (figs 3, 4 and 5).

\section{Pathological diagnoses:}

1. Recurrent lymphoma

2. Pneumocystis carinii pneumonia

3. Cholangitis

4. Cryptosporidial enteritis

5. Gelatinous marrow atrophy

6. Vacuolar myelopathy

7. Coronary artery disease

8. Disseminated atypical mycobacteriosis

9. Cytomegalovirus ependymitis

Case 2 (Dr $R$ F Miller)

A 46 year old Caucasian homosexual theatre designer presented in November 1990 with a two months history of dyspnoea, fevers and weight loss. On examination he had lymphadenopathy in the right cervical chain and crackles were audible at the right lung base. The patient requested an HIV test (as his partner was HIV-1 antibody positive); this test was positive. A chest radiograph showed diffuse bilateral shadowing and a right pleural effusion. Pleural aspirate, bronchoalveolar lavage fluid and biopsy material from a cervical lymph node all contained acid and alcohol fast bacilli (AAFB), which on subsequent culture were identified as Mycobacterium tuberculosis, thus providing an AIDS-defining diagnosis. Culture of blood, urine and stool was negative. At this time the patient's CD4 count was $340 / \mathrm{mm}^{3}$. Zidovudine was commenced and treatment for tuberculosis was begun with rifampicin, isoniazid, pyrazi- 


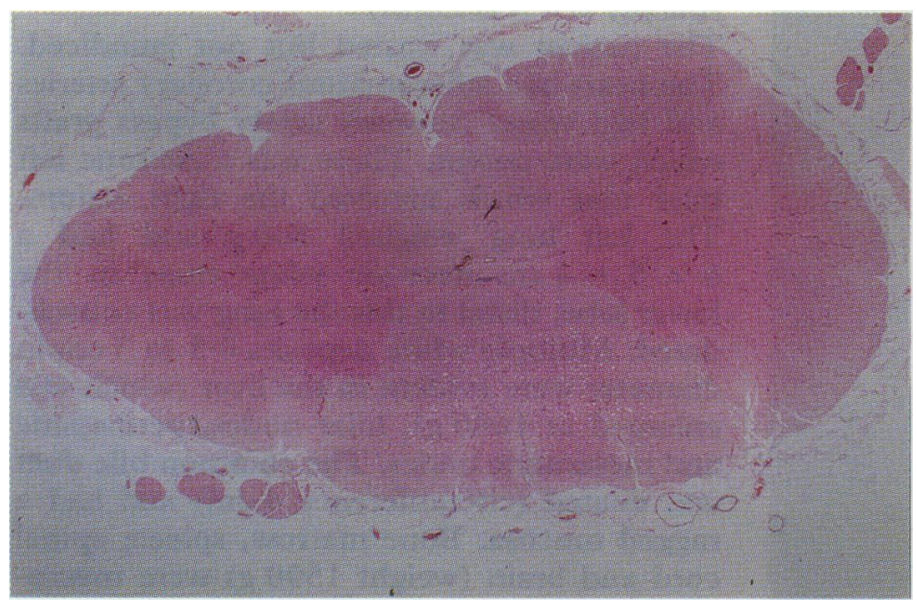

Figure 3

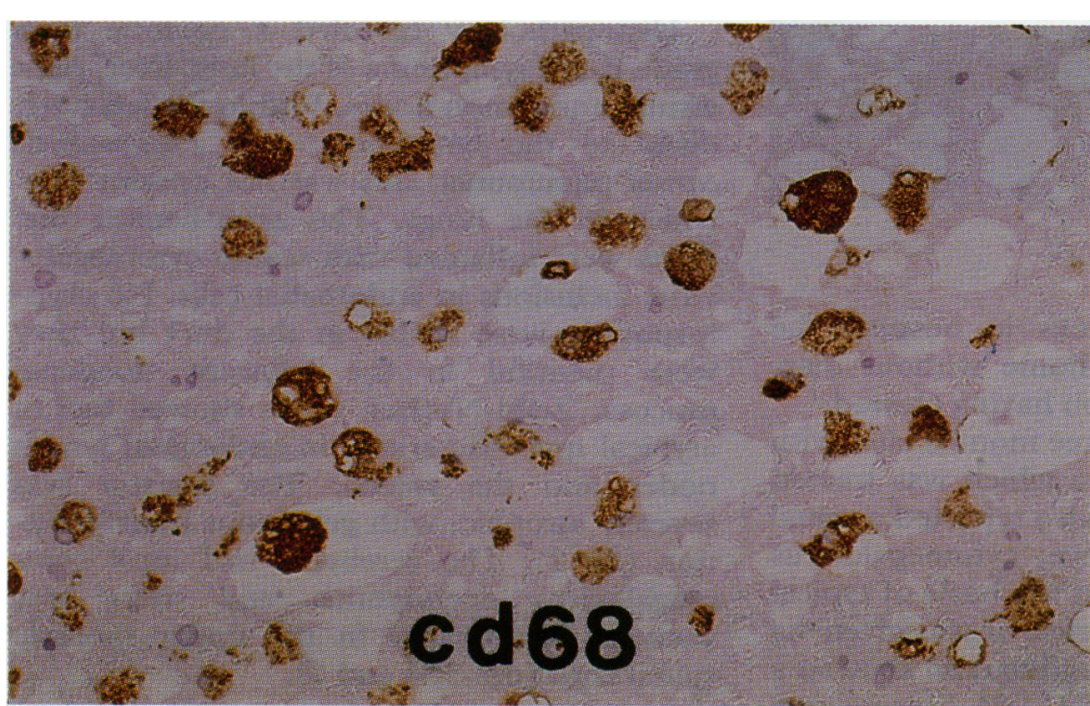

Figure 5

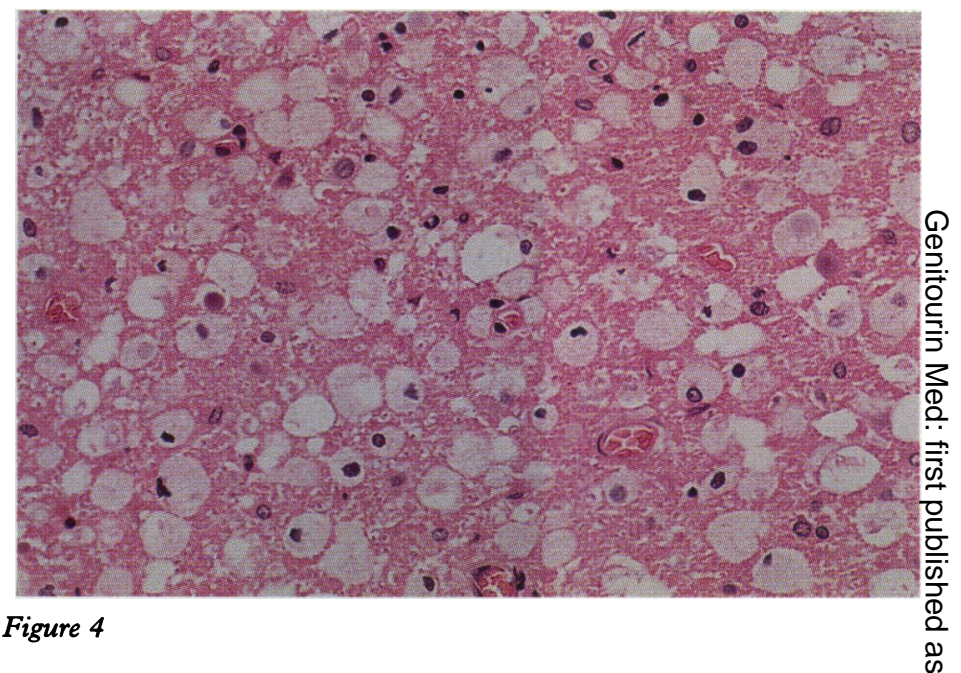

Figure 3 Case 1: Cervical spinal cord. The dorsal columns have many small holes = "aero" chocolate bar appearance. (Haematoxylin and Eosin, magnification $\times 10)$.

Figure 4 Case 1: Cervical spinal cord. The holes are vacuoles within myelin sheaths, many containing macrophages. (Haematoxylin and Eosin, magnification $\times 200)$.

Figure 5 Case 1: Cervical spinal cord. Immunostaining with CD68 to demonstrate macrophages-showing their large number and intravacuolar location. This is vacuolar myelopathy. (CD68, magnification $\times 200)$.

namide and ethambutol in conventional doses. After eight weeks of treatment antibiotic sensitivities were available and showed the organism to be fully sensitive, therefore pyrazinamide and ethambutol were stopped and rifampicin and isoniazid were continued with the aim of completing a total of twelve months treatment.

Over the next eight months the patient was monitored as an outpatient and remained well. Fluconazole was started, because of persistent oral candidiasis, and, as the CD4 count had fallen to $140 / \mathrm{mm}^{3}$, co-trimoxazole was commenced as primary prophylaxis against pneumocystis pneumonia.

$\mathrm{He}$ was admitted as an emergency in August 1991 complaining of headaches and increasing drowsiness. On examination he was pyrexial $\left(\mathrm{T}=38^{\circ} \mathrm{C}\right)$, irritable, disorientated and obtunded and had neck stiffness. Investigations showed that blood urea and serum electrolytes were normal, haemoglobin was $14.0 \mathrm{~g} / \mathrm{dl}$ and total white blood cell count was $5.0 \times 10^{9} / \mathrm{L}$ ( $70 \%$ neutrophils). Cultures of blood, urine and stool were negative and cryptococcal antigen, toxoplasma and syphilis serology were negative in blood. A CT scan of the head was normal and CSF analysis showed protein $=6.2 \mathrm{~g} / \mathrm{L}$ and 1530 cells ( $90 \%$ of which were lymphocytes) $/ \mathrm{mm}^{3}$; stain and culture were negative for bacteria, fungi and mycobacteria. An EEG showed non specific abnormalities. The diagnosis was not immediately apparent and in order to treat possible Herpes simplex encephalitis, bacterial infection and tuberculous meningitis he was given intravenous high-dose acyclovir, chlo- $\frac{3}{3}$ ramphenicol and pyrazinamide with ethambutol (in addition rifampicin and isoniazid were continued). The day after admission the $\mathrm{O}$ patient had two generalised seizures which were treated with phenytoin. At second lumbar puncture 10 days after admission CSF analysis showed a protein of $2.9 \mathrm{~g} / 1$ and a cell count of $80 / \mathrm{mm}^{3}$ (the majority being lymphocytes), culture and serology were again negative, as was DNA amplification with $M{ }^{2}$ tuberculosis-specific primers, so acyclovir and $\stackrel{\circ}{\ulcorner}$ chloramphenicol were stopped. Over the fol- $\stackrel{\Phi}{\oplus}$ lowing two weeks the patient became increasingly alert and orientated. A third lumbar puncture was performed four weeks after $\stackrel{\Phi}{\circ}$ admission, CSF protein was $2 \cdot 3 \mathrm{~g} / 1$ and cell $\stackrel{\mathbb{Q}}{\varrho}$ count was $38 / \mathrm{mm}^{3}$ (all were lymphocytes), culture and serology were again negative. In 8 view of this rifampicin, pyrazinamide and ethambutol were stopped and isoniazid was continued as secondary prophylaxis.

Shortly after this the patient suddenly developed loss of control of his legs and loss of sphincter sensation. Examination revealed a T8 sensory level. MRI of the spine, performed immediately after the onset of weakness was normal and of the head showed 
Figure 6 Case 2: MRI scan of the thoracic cord showing myelitis (arrow).

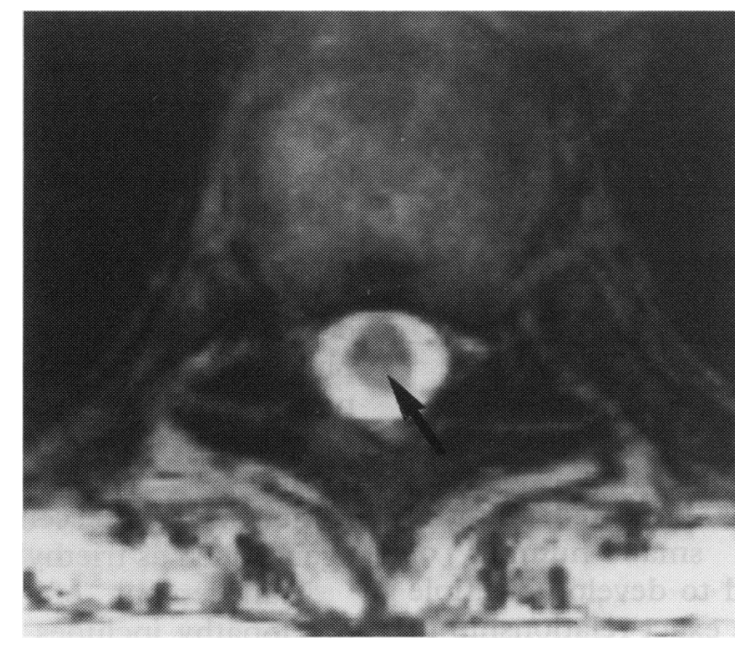

atrophy only; CSF analysis showed protein was $1 \cdot 1 \mathrm{~g} / \mathrm{l}$ and $16 \mathrm{cells} / \mathrm{mm}^{3}$ (all lymphocytes), culture and serology were negative. DNA amplification and culture for Herpes simplex and cytomegalovirus was negative. Repeat MRI of the spine performed 5 days later showed myelitis at T3 to T6 (fig 6). Empirical treatment with intravenous high dose acyclovir was given for three weeks.

The patient made a slow recovery over the next 8 weeks. Bladder and bowel sensation returned and the patient was able to stand and walk with the aid of a frame. After a period of rehabilitation he was discharged home, where he was self-caring, continent and mobile with the aid of hand rails and a wheelchair, until his death in June 1993. An autopsy was performed.

\section{Autopsy (Dr S Lucas)}

At autopsy the only external abnormality was severe wasting. The tongue showed plaques of candida. The lungs were pneumonic weighing $600 \mathrm{~g}$ (left) and $690 \mathrm{~g}$ (right). Both the spleen $(150 \mathrm{~g})$ and lymph nodes in the hilar and perihepatic sites had multiple white spots $1-5 \mathrm{~mm}$ in diameter. On cutting the brain (weight $1440 \mathrm{~g}$ ) the genu of the corpus callosum had a

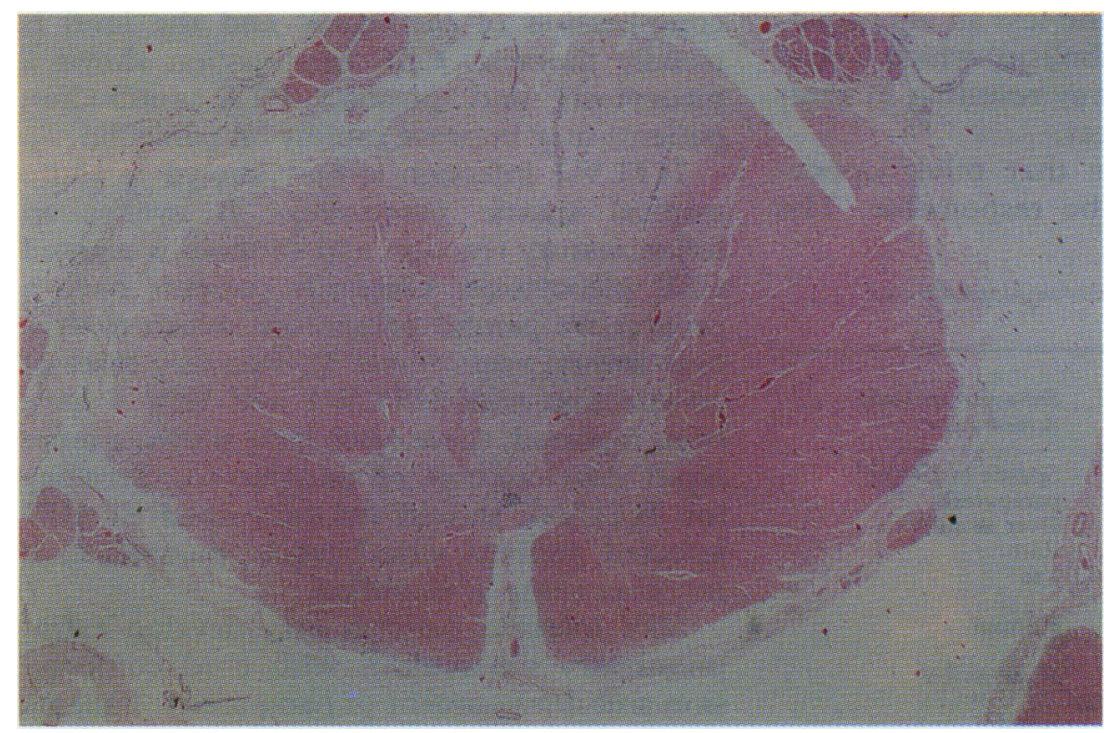

Figure 7 Case 2: Thoracic spinal cord. Vacuolar myelopathy. Massive pallor of dorsal cord and left lateral columns due to demyelination. This is more severe than in Case 1. (Haematoxylin and Eosin $\times 10)$.
$1.5 \mathrm{~cm}$ necrotic brown, partly cystic lesion. The arachnoid was adherent to the lower spinal cord and on cutting the cord it was thinned and appeared necrotic in the lower thoracic region.

Histopathology revealed bilateral candidal pneumonia and abundant candida hyphae in the tongue epithelium. The lesions in the spleen and nodes were old inactive fibrocaseous tuberculosis: Ziehl-Neelsen stains showed foci of degenerate AAFB, but cultures were negative. The lesion in the corpus callosum was severe demyelination; there were no viral inclusions. This unusual lesion resembled the process in the spinal cord, but it is not the typical site for vacuolar encephalopathy. There was a small angioma in the pons. The cord lesions were severe vacuolar myelopathy (fig 7). Immunocytochemistry and PCR analysis of the spinal tissues revealed no cytomegalovirus, $H$ simplex, Varicella zoster or JC virus.

\section{Pathological diagnoses:}

1. Severe vacuolar myelopathy

2. Candida pneumonia

3. Treated miliary tuberculosis

4. Necrosis of the corpus callosum

\section{Discussion (Professor M 9 G Harrison)}

The true incidence of spinal cord involvement in patients with HIV infection is unknown. Post-mortem studies suggest that at death the cord is abnormal in up to $47 \%$ of patients ${ }^{2}$; clinically apparent abnormalities are present in life in only $5 \%$ to $10 \% .^{3}$ This wide discrepancy has several probable explanations. Firstly, it is often difficult clinically to detect a spinal cord deficit, especially if it has no spinal sensory level, in patients who have long tract signs from HIV encephalopathy, which may co-exist. Secondly there is the problem of detecting neurological abnormalities in a bed ridden patient who is cachectic from systemic complications of HIV disease, as in the first case.

Table 1 shows the differential diagnosis of clinically apparent spinal cord disease in patients infected with HIV. At seroconversion a self-limiting temporary paraparesis, similar to acute viral myelitis in non HIV infected patients, may occur. ${ }^{4}$ Antibodies to HIV may not be present and so the diagnosis depends on the detection of HIV antiginaemia or

Table 1 Differential diagnosis of myelopathy in an HIV infected patient

\begin{tabular}{ll}
\hline Due to HIV infection & Seroconversion illness \\
& Vacuolar myelopathy \\
Due to other infections & Epidural abscess \\
& Syphilis \\
& Mycobacterium tuberculosis \\
Herpes simplex & Varicella zoster \\
& Cytomegalovirus \\
& HTLV 1 \\
& B12 \\
Due to vitamin deficiency & E \\
Due to structural causes & Lymphoma \\
& Spondylosis/disc prolapse \\
& Vascular \\
\hline
\end{tabular}


Table 2 Definition of HIV-related myelopathy

1 An acquired abnormality in the lower limbs (weakness, incoordination) which is out of proportion to upper limbs

Confirmed by clinical examination (weakness, ataxia, hyperreflexia, extensor plantars, with or without sensory hyperreflexia, extensor plantars, with or witho
changes, with or without sphincter problems)

3 Severe enough to need at least unilateral support for Severe

walking Any cognitive impairment is mild and does not affect Any cognitive impairm

No other cause identified after laboratory investigation

viraemia. Although the condition is benign, anti-retroviral therapy is indicated. Shortly after seroconversion a small number of patients have been noted to develop multiple sclerosis-like disease; the exact relationship to HIV infection is unclear.

The major cause of cord disease related to HIV infection is vacuolar myelopathy (table 2). This condition was first described in $1985^{2}$ and is usually a late complication of HIV infection, although it may be the presenting or AIDS defining condition. The clinical picture is of the development of a spastic paraparesis over the course of 3 to 16 weeks. Weakness is present in all cases, spasticity in about half, and approximately $20 \%$ have ataxia which is due to loss of joint position sense. Sphincter involvement is rare, as is a spinal level which is surprising as pathological abnormalities are most apparent in the thoracic cord (intriguingly this is also the case in patients with HTLV-1 infection and also in apes with simian AIDS). Half the patients have a coincidental peripheral neuropathy and some have dementia due to HIV encephalopathy.

The cause of vacuolar myelopathy is not known but the pathology is strikingly similar to that of vitamin $\mathrm{B} 12$ deficiency, both in terms of the involvement of posterior columns and pyramidal tracts and also in the vacuolar change (table 3 ). Although serum B12 levels are low in up to $15 \%$ of patients with AIDS, a recent study of patients with vacuolar myelopathy found no abnormality of serum B12 levels or of B12 metabolites. It has been suggested that even without evidence of B12 deficiency there is none-the-less an abnormality of methyl group metabolism ${ }^{5}$ which would suggest a common pathway resulting in similar histological appearances.

Another suggestion is that other opportunistic infections may be responsible. The

Table 3 Comparative features of HTLV-1 myelopathy, vacuolar myelopathy and subacute combined degeneration

\begin{tabular}{|c|c|c|c|}
\hline Features & $\begin{array}{l}\text { Tropical spastic } \\
\text { paraparesis/HTLV-1 } \\
\text { myelopathy }\end{array}$ & $\begin{array}{l}\text { Vacuolar } \\
\text { myelopathy }\end{array}$ & $\begin{array}{l}\text { Subacute combined } \\
\text { degeneration }\end{array}$ \\
\hline Presentation & Spastic paraparesis & Spastic paraparesis & $\begin{array}{l}\text { Spastic or flaccid } \\
\text { paraparesis }\end{array}$ \\
\hline $\begin{array}{l}\text { Onset } \\
\text { Dementia } \\
\text { Associated }\end{array}$ & $\begin{array}{l}\text { Over years } \\
\text { None } \\
\text { Yes }\end{array}$ & $\begin{array}{l}\text { Over months } \\
\text { Yes, in } \approx 60 \% \\
\text { Yes }\end{array}$ & $\begin{array}{l}\text { Over months } \\
\text { Rare } \\
\text { Yes }\end{array}$ \\
\hline $\begin{array}{l}\text { CD4 count } \\
\text { (cells } / \mathrm{mm}^{3} \text { ) }\end{array}$ & Normal & $<350$ & Normal \\
\hline $\begin{array}{l}\text { CSF cell count } \\
\left(\text { cells } / \mathrm{mm}^{3}\right)\end{array}$ & $20-100$ & None or few & None or few \\
\hline CSF antibodies & Anti HTLV-1 & Anti-HIV & None \\
\hline $\begin{array}{l}\text { Pathological } \\
\text { abnormalities }\end{array}$ & Inflammatory myelitis & $\begin{array}{l}\text { Spongy degeneration of } \\
\text { dorsal and lateral } \\
\text { columns }\end{array}$ & $\begin{array}{l}\text { Spongy degeneration of } \\
\text { dorsal and lateral } \\
\text { columns }\end{array}$ \\
\hline
\end{tabular}

main evidence for this hypothesis, as no infection has been directly identified, is that vacuolar myelopathy is rare in HIV infected children (occurring in about $2 \%$ ) and children have had little chance to acquire opportunistic infective agents. A third suggestion is that vacuolar myelopathy is a direct effect of HIV infection itself. HIV is rarely detected in the cord, the exception being within macrophages. It is possible that activated macrophages release cytokines which are then responsible for the intramyelin oedema and splitting, pathological changes which are somewhat reminiscent of the toxic effects of agents such as triethyltin.

Management of patients with vacuolar myelopathy includes imaging of the cord, to exclude rival causes of cord disease. Treatment is usually limited to using drugs such as baclofen for spacticity, and physiotherapy to maintain mobility. Anti retroviral drugs might be prescribed if the patient was not already receiving them; in some centres empirical B12 injections might be given.

Other infections that can cause spinal cord disease in HIV infected patients include pyogenic epidural abscesses. These are rare, but their incidence is increasing in the intravenous drug using population, due to the frequent occurrence of bacteraemia. In this setting patients present with fever, back pain and root pain and the rapid development of parparesis over 48 to 72 hours. Plain radiology and CT may show osteomyelitis but MRI best demonstrates the lesions. If the patient also has meningitis, gadolinium enhancement may occur and so confuse assessment of the cord lesion; in this situation myelography would be the preferred investigation. CSF examination may be hazardous and cultures are only positive in $20 \%$, so this investigation is not carried out routinely. Despite early intervention with antibiotics surgery may be necessary to decompress the cord and establish an aetiological diagnosis.

Syphilis may also cause an acute or subacute paraparesis, with bladder or bowel disturbance, and a sensory level. This occurs as a complication of meningitis and the level is usually thoracic. CSF examination shows a pleocytosis and positive FTA antibodies; patients may improve rapidly on penicillin.

HTLV-1 infection is now known to cause tropical spastic paraparesis. It evokes an inflammatory reaction and so there is marked CSF pleocytosis. Clinically patients have a progressive painful paraparesis which evolves over several years (table 3 ). Recently cases of co-infection with HTLV-1 and HIV 1 have been reported; these cases have shown a more rapid development of paraparesis. Serology for HTLV-1 should be performed in HIV infected patients who become paraplegic, if no other cause is identified.

HIV infected patients may develop extrapulmonary tuberculosis with cord compression and development of paraparesis. Patients usually have CD4 counts $<250 / \mathrm{mm}^{3}$ and the paraparesis often occurs on the background of a meningitis. 
Rarely Herpes zoster ${ }^{2}$ infection may be complicated by a myelitis one to three weeks after the shingles. An ipsilateral motor weakness may develop, followed by spinothalamic then posterior column involvement leading to a paraparesis or quadriparesis. ${ }^{2}$ The diagnosis is suggested by the typical dermatomal skin rash and inflammatory CSF with gadolinium enhancement at the appropriate level, on MRI of the cord. Varicella zoster virus may be cultured from CSF, alternatively it may be detected using the polymerase chain reaction. ${ }^{6}$

Cytomegalovirus infection may cause rapidly developing spinal cord lesions in HIV infected patients with CD4 counts below $50 / \mathrm{mm}^{3}$. A CSF pleocytosis is typical and virus may be detected by culture or PCR. More usually cytomegalovirus causes a radiculopathy ${ }^{7} ; \mathrm{MRI}$ with gadolinium shows enhancement of the roots and cauda equina. Cytomegalovirus myelitis should be considered if a patient develops a spinal cord lesion of rapid onset and has cytomegalovirus retinitis or viraemia; treatment is with intravenous ganciclovir. This clinical picture was apparent in the second case and was suggestive of cytomegalovirus. Herpes simplex virus may cause a similarly rapid onset myelitis but in this infection CSF pleocytosis is much less marked; diagnosis relies on culture or PCR of virus from CSF. Usually Herpes simplex infection occurs as a co-infection with cytomegalovirus; treatment is with intravenous high-dose acyclovir.

Structural disease of the spinal cord must always be considered and masses may be due to abscess, lymphoma, plasmacytoma or glioma. Patients usually present with spinal pain and then develop paraplegia. Imaging of the spine with CT or MR reveals a mass lesion. Surgery is usually necessary to make a tissue diagnosis.

Vascular disease may rarely cause spinal cord involvement. Haemorrhage into the cord may occur in thrombocytopenic patients. Cord ischaemia may be suspected if there is sudden onset of paraparesis with preservation of joint position sense. This phenomenon occurs due to anterior spinal artery occlusion, with sparing of the posterior third of the cord. Only the sudden onset discriminates this from cord compression caused by lymphoma. In this way the presentation of the second case could have been vascular in origin.

When it is clear that a spinal cord lesion has developed in an HIV infected patient, investigation will normally require imaging with CT or $\mathrm{MR}$ to exclude a treatable cause of cord compression, such as epidural abscess or lymphoma. A dramatically sudden onset suggests a vascular cause, whilst development of a paraparesis over a few days, or a week, raises the possibility of CMV or herpes virus infection. An insidious onset of weakness over many weeks or months is most likely due to vacuolar myelopathy. The two cases presented illustrate the range of clinically apparent spinal cord disease due to this process.

1 Fuller GN, Jacobs JM, Guiloff RJ. Subclinical periphera nerve involvement in AIDS: an electrophysiological and neuropathological study. $₹$ Neurol Neurosurg Psychiatry 1991;54:318-24

2 Petito CK, Navia BA, Cho ES, Jordan BD, et al. Vacuola myelopathy pathologically resembling subacute combined degeneration in patients with the acquired immune deficiency syndrome. $N$ Engl f Med 1985;312. 874-9.

3 McArthur JC. Neurologic manifestations of AIDS Medicine (Baltimore) 1987:66:407-37.

4 Denning DW, Anderson J, Rudge P, Smith H. Acute myelopathy associated with primary infection with myelopathy associated with primary infection with immunodeficiency virus. $B M \Im 1987 ; \mathbf{1}: 143-4$.

5 Surtees R, Hyland K, Smith I. Central nervous system methyl group metabolism in children with neurological methyl group metabolism in children with neurologica

6 Grant AD, Fox JD, Brink NS, Miller RF. Detection of varicella-zoster virus DNA using the polymerase chain reaction in an immunocompromised patient with trans verse myelitis secondary to Herpes zoster. Genitourin Med 1993;69:273-5.

7 Eidelberg D, Sotrel A, Vogel H, et al. Progressive polyradiculopathy in acquired immune deficiency syndrome. Neurology 1986;36:912-6. 